\title{
La pericolosità sociale fra neuroscienze, misure di sicurezza detentive e nuove prospettive introdotte dalla Legge n. 81 del 2014
}

\author{
Francesca SAMPERI ${ }^{1}$, Alessandro SANTURRO ${ }^{1}$ *, Susanna AGOSTINI ${ }^{1}$, \\ Mariantonia DI SANZO 1, Alessandro MARIANI ${ }^{1}$, Mauro ARCANGELI ${ }^{2}$
}

1Dipartimento di Scienze Anatomiche, Istologiche, Medico Legali e dell'Apparato Locomotore, Sapienza Università di Roma, Roma, Italia; ${ }^{2}$ Dipartimento di Medicina Interna e Sanità Pubblica, Università degli Studi dell'Aquila, L'Aquila, Italia

*Corresponding author: Alessandro Santurro, Dipartimento di Scienze Anatomiche, Istologiche, Medico Legali e dell'Apparato Locomotore, Sapienza - Università di Roma, Roma, Italia. E-mail: alessandro.santurro@uniroma1.it

\section{A B S T R A C T}

SOCIAL DANGEROUSNESS BETWEEN NEUROSCIENCES, ADMINISTRATIVE DETENTION AND NEW PERSPECTIVES INTRODUCED BY LAW No. 81/2014

With regard to dangerous individuals, the Italian legal system has two main tools: preliminary injunction and imprisonment. The latter aims at rehabilitating the detainee while at the same time safeguarding the society. Law No. 81/2014 has deeply affected this issue, making it more topical than ever, by closing Judicial Psychiatric Hospitals and replacing them with the so-called Residenze per l'Esecuzione delle Misure di Sicurezza (REMS), in an attempt to create a regulatory system which limits the resort to detention as much as possible, confining it to a subsidiary role.

(Cite this article as: Samperi F, Santurro A, Agostini S, Di Sanzo M, Mariani A, Arcangeli M. La pericolosità sociale fra neuroscienze, misure di sicurezza detentive e nuove prospettive introdotte dalla Legge n. 81 del 2014. Minerva Medicoleg 2016;

Key words: Dangerous behavior - Neurosciences - Forensic psychiatry - Insanity defense. 66 Premetto che all'espressione di perico-
losità si collega il suo vero e preciso significato e che con essa null'altro si intende se non quella caratteristica della persona che con probabilità ed effettivamente violerà $i$ diritti" 1 . Tale concetto di pericolosità, frutto di una lenta e complessa evoluzione della scienza penale, cominciò a essere compiutamente definito quando fu posto al centro della polemica che, tra la fine del XIX la prima metà del XX secolo, animò il dibattito fra la Scuola Classica, quale sostenitrice di un'idea di responsabilità fondata sulla capacità di volere e quindi sul libero arbitrio, e la Scuola Positiva del diritto penale, quale sostenitrice, invece, di un'idea di criminale frutto di un insieme di fattori sia di tipo biologico e anatomico, che di tipo sociologico e ambientale.

La nozione di pericolosità sociale (dell'individuo autore di un fatto preveduto dalla legge come reato) e le tipologie soggettive del delinquente fecero ingresso nell'ordinamento giuridico italiano con il Codice Rocco (1930), quali presupposti delle misure di sicurezza, che, insieme alle pene, concorrono tuttora a costituire il modello che è stato definito del 
"doppio binario". Mentre, infatti, veniva mantenuta l'idea di una responsabilità individuale cui si correla la pena, veniva anche introdotto il concetto di pericolosità, al quale corrisponde la misura di sicurezza. L'art. 203 del codice penale stabilisce a questo proposito che "agli effetti della legge penale è socialmente pericolosa la persona anche se non imputabile o non punibile, la quale ha commesso taluni dei fatti indicati nell'articolo precedente《sup» ${ }^{\mathrm{i}}\langle/$ sup», quando è probabile che commetta nuovi fatti preveduti dalla legge come reati. La qualità di persona socialmente pericolosa si desume dalle circostanze nell'art. 133". Il c.p. del 1930, nel suo assetto originario, fissava, all'art. 204, $1^{\circ}$ comma, la regola dell'accertamento in concreto《sup» ${ }^{i i} \ll /$ sup», utilizzando al tal fine le circostanze indicate nell'art. 133 c.p. Tuttavia, a tale regola si contrapponeva subito l'eccezione, prevista dal $2^{\circ}$ comma dell'art. 204 c.p.«sup» ${ }^{i i i} \ll /$ sup».

Nella realtà del sistema, il rapporto da regola a eccezione risultava tuttavia letteralmente invertito, nel senso che, mentre il ricorso a presunzioni, in termini di frequenza, costituiva la regola, l'accertamento giudiziale risultava del tutto marginale. Tale impostazione comportava che, inevitabilmente, il giudice si trovasse nelle condizioni di doversi avvalere di valutazioni astratte, la cui ragionevolezza ed effettiva veridicità erano spesso smentite dai riscontri reali.

Nel 1983 la Corte costituzionale si è misurata con un altro profilo delle presunzioni di pericolosità rappresentato dalla persistenza temporale, pur essa presunta, dei fattori indizianti posti a fondamento della fattispecie sintomatica. L'art 204, $2^{\circ}$ comma c.p. stabiliva, infatti, la necessità che, nel caso di condanna o di proscioglimento pronunciati dopo un certo periodo dalla commissione del fatto,

\footnotetext{
i Art. 202 c.p.: "Le misure di sicurezza possono essere applicate soltanto alle persone socialmente pericolose, che abbiano commesso un fatto preveduto dalla legge come reato. La legge penale determina i casi nei quali a persone socialmente pericolose possono essere applicate misure di sicurezza per un fatto non preveduto dalla legge come reato".

ii Art 204, $1^{\circ}$ comma c.p.: "Le misure di sicurezza sono ordinate, previo accertamento che colui il quale ha commesso il fatto è persona socialmente pericolosa".

iii Art 204, $2^{\circ}$ comma c.p.: "Nei casi espressamente determinati, la qualità di persona socialmente pericolosa è presunta dalla legge".
}

l'applicazione della misura di sicurezza fosse comunque subordinata all'accertamento giudiziale della pericolosità. "Senonché, in taluni casi, tuttavia, (ed in particolare nell'ipotesi di assegnazione a una casa di cura e custodia del condannato a pena diminuita a causa di infermità psichica, ex art. $219,1^{\circ}$ e $2^{\circ} \mathrm{com}-$ ma c.p., e nell'ipotesi di ricovero in ospedale psichiatrico giudiziario del prosciolto per infermità psichica, ex art. 222 c.p.), l'elemento indiziante di carattere personale, rappresentato per l'appunto dall'infermità psichica, viene accertato in riferimento alla commissione del fatto. Questa, tuttavia, precede, e spesso di molto, l'applicazione della misura di sicurezza, che potrebbe pertanto essere adottata in un momento in cui non v'è alcuna certezza circa l'attuale sussistenza del fattore indiziante: il prosciolto o il condannato a pena diminuita può infatti, nel frattempo, essere guarito dall'infermità psichica che lo aveva colpito e che sussisteva al momento della condotta criminosa" 2 .

D'altro canto l'accertamento di un' infermità psichica attuale non presenta, in effetti, difficoltà maggiori di quelle insite nell'analogo accertamento svolto in rapporto alla commissione del fatto; anzi si presenta più agevole, proprio perché non implica alcuna "trasposizione personale" del soggetto.

Queste ragioni hanno indotto la Corte Costituzionale 3,4 a dichiarare l'illegittimità degli artt. 222 e $219,1^{\circ}$ e $2^{\circ}$ comma c.p. nella parte in cui essi non subordinano il provvedimento di applicazione della misura "al previo accertamento da parte del giudice della persistente pericolosità sociale derivante dall'infermità [...] al tempo dell'applicazione della misura di sicurezza".

Infine, l'art. 31, $1^{\circ}$ comma, L. 663/1986, abrogando espressamente l'art. 204 c.p., ha eliminato dal sistema le presunzioni di pericolosità. A seguito di questo intervento legislativo, l'assetto normativo della pericolosità (e delle misure di sicurezza a essa correlate) si è sostanzialmente trasformato, ottenendo, quindi, un sistema interamente ed esclusivamente imperniato sull'accertamento giudiziale in concreto $^{5}$. 


\section{Imputabilità e neuroscienze nella giurisprudenza penale italiana}

L'istituto della pericolosità sociale, come accennato, insieme alla pena, costituisce il cosiddetto sistema del "doppio binario", in cui la pena si pone quale risposta all'azione criminosa di un soggetto ritenuto imputabile, e l'applicazione della misura di sicurezza, il cui presupposto è appunto la pericolosità sociale, quale risposta alla commissione di un reato da parte di un soggetto ritenuto non imputabile o parzialmente tale, o eventualmente anche applicata in aggiunta alla pena stessa nei casi in cui risulti necessario. Il concetto di pericolosità sociale è inevitabilmente, quindi, legato a quello dell'imputabilità, la quale oggi, grazie all'ausilio di innovativi studi scientifici può essere verificata con nuovi strumenti. Il tema dell'accertamento dell'imputabilità in caso di infermità mentale è, infatti, sempre più spesso oggetto di dibattiti dottrinali e giurisprudenziali, costituendo uno degli esempi più emblematici dell'interazione tra Scienza e Diritto.

Le Sezioni Unite, con la pronuncia n. 9163 dell'8.3.2005, conosciuta come sentenza Raso ${ }^{6}$, hanno chiarito che nella nozione di infermità penalmente rilevante possono essere ricompresi anche $i$ disturbi della personalità. Questi, quindi, possono costituire causa di esclusione o di limitazione dell'imputabilità, a condizione, però, "che il giudice ne accerti la gravità e l'intensità, tali da escludere o scemare grandemente la capacita di intendere $e$ di volere, e il nesso eziologico con la specifica azione criminosa" 7. Le Sezioni Unite hanno innanzitutto rilevato che "la imputabilità, che si sostanzia nella capacità di intendere e di volere, non è solo una mera condizione soggettiva di riferibilità della pena, cioè una 'mera capacità di pena', ma è invece 'capacità di reato' ovvero 'capacità di colpevolezza', che racchiude in sé l'assioma che non può esservi (non già solo pena, ma ancora prima) consapevolezza senza imputabilità. Da qui il passaggio successivo: non può esservi colpevolezza senza rimproverabilità, la quale postula, a sua volta, 'la necessità, per la punibilità del reato, della effettiva coscienza, nell'agente, della an- tigiuridicità del fatto" " 8 . E nel commentare la sentenza, la dottrina ha avuto modo di rilevare che "la Corte, sensibile agli orientamenti più moderni sia della scienza psicopatologica che della scienza penalistica, valorizza a fondo il modello a due piani del giudizio di imputabilità (giudizio misto o biologico-normativo)" 9; e che "assume un altissimo valore, per le future applicazioni concrete della normativa in materia di imputabilità e per lo stesso procedimento ermeneutico utilizzato, il richiamo, contenuto nella sentenza alla necessaria collaborazione tra giustizia penale e scienza" 10 .

Le tecniche neuroscientifiche possono fornire un grande contributo relativamente a questo aspetto specifico; oggi, infatti, è possibile evidenziare quale sia il correlato neurale, o i correlati neurali, responsabile del disturbo di personalità. "Il grave disturbo di personalità ha infatti maggiori probabilità di avere dei correlati microstrutturali evidenziabili alla $V B M$, mentre il disturbo di personalità lieve non presenta questa caratteristica. In altri termini esiste un aumento proporzionale dell'alterazione microstrutturale in funzione dell'intensità con cui si manifesta la sintomatologia e il transito da intensità lieve a intensità media e grave della sintomatologia è quindi accompagnato dall'alterazione microstrutturale evidenziabile alla VBM«sup» iv «/sup»" 11.

Negli ultimi anni è ormai possibile, attraver-


struttura cerebrale e quantificarne la funzionalità, individuando le alterazioni strutturali e i fenomeni elettro-chimici delle aree encefaliche sottese al controllo emotivo e comportamentale, consentendo lo studio diretto dell'attività cerebrale messa alla prova da una stimolazione emotiva o durante la risposta comportamentale

\footnotetext{
iv La morfometria basata sui voxel (VBM) è una tecnica di analisi in neuroimaging che consiste nella ricerca di differenze focali nell'anatomia del cervello, usando l'approccio statistico noto come mappatura statistica parametrica.

v Sono diversi gli strumenti di visualizzazione cerebrale (brain imaging) di cui attualmente disponiamo, tra cui: 1'analisi computerizzata del tracciato EEG, che realizza un mappaggio selettivo dell'attività elettrica di specifiche aree cerebrali, la tomografia assiale computerizzata (TAC), la risonanza magnetica funzionale (fMRI), la tomografia ad emissione di positroni (PET), la magnetoencefalografia (MEG), la tomografia computerizzata ed emissionale di fotoni singoli (SPECT) nonché le acquisizioni sull'attività neurotrasmettitoriale e neuromodulatoria, fino allo studio della neurobiologia molecolare, ecc.
} 
in condizioni fisiologiche, o in particolari condizioni patologiche ${ }^{12-17}$. Varianti genetiche riguardanti i neuro-modulatori chiave coinvolti nel controllo dell'aggressività (in particolare, serotonina, dopamina, steroidi sessuali, glucocorticoidi e arginina-vasopressina) rappresentano dei possibili marcatori biologici della predisposizione verso condotte criminali violente. Numerosi studi hanno investigato i geni coinvolti nelle vie molecolari dei neuromodulatori, ma i risultati ottenuti sono stati per lo più contraddittori o non replicati ${ }^{18-22}$. Solo alcuni geni sono stati associati con condotte impulsivo-aggressive in modo piuttosto univoco (SLC6A4 e MAOA, che hanno un ruolo chiave nel controllo della neurotrasmissione serotoninergica cerebrale) ${ }^{23-24}$, ma la varianza è stata stimata non superiore al 5\% per la variante genetica con la maggior evidenza di associazione (5-HTTLPR) ${ }^{25}$. I risultati descritti riflettono la complessità di tratti comportamentali quali aggressività ed impulsività, $\mathrm{i}$ quali sono caratterizzati da una labilità biologica legata a numerose varianti genetiche, ma risentono in larga misura anche dell'influenza dell'ambiente (specialmente durante l'infanzia). Da ciò discende che "un approccio integrato comprendente variabili biologiche, psicologiche e sociali appare il più appropriato all'indagine del comportamento motivato umano, anche se il rispettivo contributo dei citati fattori e la metodologia piu appropriata per la loro indagine sono tutt'oggi oggetto di dibattito" 26 . In tale contesto, le cosiddette neuroscienze sociali, ad esempio, indagano i correlati neurali legali alla capacità di un individuo di mantenere un comportamento adeguato in un determinato contesto sociale, la capacità di interpretare gli stati emotivi propri e altrui, il giudizio morale, la capacità di autocontrollo, e, in generale, l'empatia.

D'altra parte la rilevanza dei metodi neuroscientifici, così come è stata recepita nella pratica del processo penale, può essere rilevata nelle sentenze di Cassazione n. 43786/2010, Cassazione n. 36080/2015, Corte d'Assise d'Appello di Trieste n. 5/2009, Tribunale di Como n. 536/2011 e Tribunale di Cremona $n$. 109/2011.
Con la sentenza n. 43786 del 2010, la Corte di Cassazione si rifà alla sentenza Daubert, con cui la Corte Suprema degli Stati Uniti d'America, nel 1993, ha individuato come "criteri per l'apprezzamento della scienza valida (ossia rilevante e affidabile) la possibilità di verificare e falsificare il principio scientifico da impiegare; che esso sia stato oggetto di una revisione paritaria da parte dei componenti della comunità scientifica; che i risultati delle ricerche siano stati pubblicati in riviste specialistiche; che sia considerata la percentuale di errore, nota o potenziale, della teoria scientifica; che siano rispettati gli standard di corretta esecuzione delle operazioni applicative inerenti quel determinato principio scientifico; che vi sia stata accettazione della teoria nella comunità scientifica" ${ }^{27}$. La Corte di Cassazione, con la sentenza n. 43786 del 2010, ha ritenuto, quindi, che per valutare l'attendibilità di una teoria occorre esaminare gli studi che la sorreggono, "le basi fattuali sui quali essi sono condotti, l'ampiezza, la rigorosità, l'oggettività della ricerca. Il grado di sostegno che i fatti accordano alla tesi. La discussione critica che ha accompagnato l'elaborazione dello studio, focalizzata sia sui fatti che mettono in discussione l'ipotesi sia sulle diverse opinioni che nel corso della discussione si sono formate. L'attitudine esplicativa dell'elaborazione teorica. Ancora, rileva il grado di consenso che la tesi raccoglie nella comunità scientifica. Infine, dal punto di vista del giudice, che risolve casi ed esamina conflitti aspri, è di preminente rilievo l'identità, l'autorità indiscussa, l'indipendenza del soggetto che gestisce la ricerca, le finalità per cui si muove" 28.

Concetti molto simili sono stati ribaditi dalla Cassazione con la sentenza n. 36080 del 2015, in cui si afferma: "un risultato di prova scientifica può essere ritenuto attendibile solo ove sia controllato dal giudice, quantomeno con riferimento all 'attendibilità soggettiva di chi lo sostenga, alla scientificità del metodo adoperato, al margine di errore più o meno accettabile ed all'obiettiva valenza ed attendibilità del risultato conseguito. Insomma, secondo un metodo di approccio critico non dissimile, concettualmente, da quello richiesto 
per l'apprezzamento delle prove ordinarie, al fine di esaltare, quanto più possibile, il grado di affidabilità della 'verità processuale'o - se si preferisce - ridurre a margini ragionevoli l'ineludibile scarto tra verità processuale e verità sostanziale" 29.

Unitamente alla Corte di Cassazione, che ha gettato le basi in materia di attendibilità delle prove scientifiche e, in generale, del metodo scientifico quale mezzo per la ricerca delle stesse, la sentenza n. 5/2009 della Corte d'Assise d'Appello di Trieste, per la prima volta in Italia, ha preso in considerazione i risultati emersi da indagini di genetica molecolare e da tecniche neuropsicologiche, al fine di determinare il grado di incapacità di intendere e di volere dell'imputato ${ }^{30}$.

Il caso riguarda un omicidio commesso da un algerino, seguito dal Centro di Salute Mentale, a danno di un colombiano, erroneamente scambiato dall'imputato per il responsabile di un'aggressione da lui precedentemente subita. La Corte d'Assise d'Appello di Trieste aveva conferito l'incarico peritale, richiedendo di effettuare le valutazioni necessarie sulla capacità di intendere e di volere dell'imputato al momento dei fatti, sull'eventuale presenza di patologie psichiatriche e sul suo grado di pericolosità sociale. Le conclusioni alle quali erano pervenuti i periti dimostravano come "[l'imputato] presentava all'epoca dei fatti una infermità di mente avendo egli una patologia psichiatrica documentata e grave [...] lo stato psichico [dell'imputato] impediva allo stesso di comprendere pienamente il comportamento in una interazione sociale [l'imputato presenta] un quadro psichiatrico caratterizzato da una tipologia di personalità di tipo 'dipendente-negativistico' con un importante disturbo ansioso-depressivo accompagnata da pensieri deliranti ed alterazioni del pensiero [...] associato a deficit cognitivi [...] Per quanto riguarda la capacità di volere questa è grandemente scemata ma non abolita [...] [L'imputato] è attualmente da considerarsi pericoloso socialmente non essendo stati pienamente rimossi $i$ fattori causativi del reato (psicopatologia)". La Corte, pertanto, riteneva di dover fare proprie le conclusioni cui era- no pervenuti i periti. "Le indagini svolte dai periti (diagnosi descrittiva, diagnosi di sede, diagnosi di natura) - si sono dimostrate particolarmente accurate ed immuni da illogicità sul piano procedimentale o di argomentazioni antinomiche. Esse restituiscono un quadro coerente e credibile della condizione mentale dell'imputato concludendo per la sua parziale incapacità d'intendere e di volere". La Corte, inoltre, riponeva particolare affidamento nelle tecniche utilizzate dai periti, tra le quali diversi test neuropsicologici, la risonanza magnetica cerebrale e l'analisi del genotipo, accogliendo in particolare quest'ultima e mettendone in evidenza la novità: "Particolarmente significative sono risultate le indagini genetiche effettuate dai periti alla ricerca di polimorfismi genetici significativi per modulare le reazioni a variabili ambientali fra $i$ quali in particolare per quello che interessa nel caso di specie l'esposizione ad eventi stressanti ed a reagire agli stessi con comportamenti di tipo impulsivo". Tale indagine, del tutto innovativa rispetto al livello di approfondimento corrente degli accertamenti giudiziari, avrebbe consentito di accertare che 1'imputato "risulta possedere, per ciascuno dei polimorfismi esaminati, almeno uno se non tutti e due gli alleli che, in base a numerosi studi internazionali riportati sinora in letteratura, sono stati riscontrati conferire un significativo aumento del rischio di sviluppo di comportamento aggressivo, impulsivo (socialmente inaccettabile)". Il soggetto con tale profilo genetico sarebbe stato quindi "maggiormente incline a manifestare aggressività se provocato o escluso socialmente. È opportuno sottolineare che tale 'vulnerabilità genetica' risulta avere un peso ancor più significativo nel caso in cui l'individuo sia cresciuto in un contesto familiare e sociale non positivo e sia stato, specialmente nelle prime decadi della vita, esposto a fattori ambientali sfavorevoli, psicologicamente traumatici o negativi'. Proprio l'interazione tra geni ed ambiente è stato l'elemento che ha assunto un ruolo preponderante nella decisione dei Giudici. Infatti, "proprio la circostanza emersa nel corso dell'ultima perizia psichiatrica e, vale a dire, che determinati 'geni' presenti nel 
patrimonio cromosomico dell'imputato lo renderebbero particolarmente reattivo in termini di aggressività (e, conseguentemente, vulnerabile) in presenza di situazioni di stress, induce la Corte a rivalutare la decisione del G.U.P. di non applicare al massimo la riduzione di pena possibile per il difetto parziale di imputabilità. Proprio l'importanza del deficit riscontrato dai periti con queste nuovissime risultanze frutto dell'indagine genetica portano a ritenere che la riduzione possa essere effettivamente operata nella misura massima di un terzo".

È importante sottolineare come il concetto di vulnerabilità genetica sia stato assunto come spiegazione remota del comportamento aggressivo messo in atto e non come giustificazione di esso. Se, da un lato, la Corte osservava che le differenze culturali e la fede religiosa professata non avrebbero potuto in ogni caso costituire un fondamento giustificativo per un'aggressione a fini omicidi, dall'altro in nessun luogo si affermava che la predisposizione genetica potesse giustificare il comportamento aggressivo. A tal proposito si osservi, anzi, come il giudizio di pericolosità sociale dell'imputato sia rimasto inalterato rispetto alla sentenza di primo grado. La Corte, condividendo anche in questo caso le conclusioni dei periti, riteneva che l'imputato fosse da considerarsi pericoloso socialmente 31 .

La sentenza del Tribunale di Como n. 536 del 20 maggio 2011 si riferisce all'omicidio commesso da una donna ai danni della sorella maggiore, precedentemente sequestrata in casa e costretta ad assumere psicofarmaci in dosi tali da indurla in uno stato di confusione mentale e di incapacità reattiva; successivamente la donna aveva tentato di occultarne il cadavere dandolo alle fiamme. L'imputata veniva pertanto chiamata a rispondere di sequestro di persona e omicidio, nonché dei reati di soppressione e distruzione di cadavere, utilizzo indebito delle carte di credito appartenenti alla sorella, e ancora di procurata incapacità di intendere e di volere del padre attraverso la somministrazione di medicinali che ne procurarono il ricovero in ospedale, di tentato omicidio di entrambi i genitori, avendo cercato di farne esplodere l'autovettura, e del tentato omicidio della madre mediante strangolamento durante un diverbio. Il GIP condannava l'imputata a venti anni di reclusione, riconoscendole un vizio parziale di mente per la presenza di alterazioni in un'area del cervello che ha la funzione di regolare le azioni aggressive e, dal punto di vista genetico, di fattori significativamente associati ad un maggior rischio di comportamento impulsivo, aggressivo e violento. La decisione veniva supportata oltre che da accertamenti psichiatrici tradizionali, anche da analisi neuroscientifiche, che rivelavano la morfologia cerebrale e il patrimonio genetico dell'imputata. Si trattava, pertanto, del primo riconoscimento in Italia, e fra i primi al mondo, della validità delle neuroscienze per l'accertamento dell'imputabilità. La perizia psichiatrica aveva già riconosciuto nella donna la presenza di "un quadro psichiatrico caratterizzato dalla menzogna patologica" e di una sindrome dissociativa. Ma il GIP si era basato prevalentemente su una consulenza neuro-scientifica, che era stata richiesta dalla difesa dell'imputata. In particolare, si era proceduto alla "ricostruzione del correlato anatomo-funzionale della sfera psichica della paziente attraverso le indagini di imaging cerebrale e di genetica molecolare". Ne era emerso il riscontro di "anomalie che si traducono in un significativo aumento del rischio di sviluppare certi tipi di comportamenti". Nello specifico, i periti avevano evidenziato delle "differenze nella morfologia e nel volume delle strutture cerebrali [...] alterazioni nella densità della sostanza grigia, in alcune zone chiave del cervello [...] anche nei processi che regolano la menzogna, oltre che nei processi di suggestionabilità ed autosuggestionabilità e nella regolazione delle azioni aggressive". Infine, "sono stati, disposti accertamenti genetici per verificare se l'imputata presentasse gli alleli che, secondo la letteratura scientifica internazionale, sono significativamente associati ad un maggior rischio di comportamento impulsivo, aggressivo e violento". L'esito positivo di tali analisi (era stata riscontrata l'esistenza nell'imputata di tre alleli sfavorevoli) aveva poi portato alla decisione a favore del vizio parziale di mente. Gli esiti delle indagini di imaging cerebrale e di 
genetica molecolare erano apparsi particolarmente significativi, costituendo degli elementi ulteriori da porre a confronto con quelli della diagnosi classica. Le anomalie riscontrate con tecnica VBM, avevano evidenziato nell'imputata un aumento del rischio di sviluppare certi comportamenti, dato avvalorato da rilevamenti genetici. Sulla base dei risultati ottenuti, i periti della difesa avevano pertanto sostenuto la mancanza di una piena capacità di controllo delle proprie azioni, di percepirne il disvalore e l'assenza, quindi, di una libera autodeterminazione. Il giudice, quindi, sulla base di queste argomentazioni, e sulla base degli esiti delle indagini scientifiche svolte, riteneva che le problematiche psichiatriche riscontrate erano state parzialmente determinanti ai fini dei crimini commessi, e dunque riteneva che la capacità critica e di controllo non fosse piena al momento del fatto 32 .

"Le neuroscienze e la genetica sembrerebbero dunque, offrire un'evidenza obbiettiva in grado di circoscrivere gli ampi margini di discrezionalità insiti nei tradizionali metodi di indagine. Come sottolineato dallo stesso giudice, non si tratta, però, di introdurre nel processo penale una rivoluzione copernicana in tema di accertamento, valutazione e diagnosi delle patologie mentali né, tantomeno, di introdurre criteri deterministici da cui inferire automaticamente che ad una certa alterazione morfologica del cervello conseguono certi comportamenti e non altri', bensi di ricercare le possibili correlazioni tra le anomalie presentate da certe aree del cervello, oppure la presenza di determinati alleli di geni e il rischio di sviluppare comportamenti aggressivi, di discontrollo dell'impulsività, ovvero comportamenti socialmente inaccettabili. In buona sostanza, si giunge a un quadro più preciso dell'infermità di mente dell'imputato, ma non a giustificare deterministicamente la causazione di un reato. Ciò che, infatti, resta da valutare è se il comportamento criminoso sia il frutto di una specifica patologia" 33.

Da ultimo, la sentenza del Tribunale di Cremona, n. 109 del 19 luglio 2011, si pone come importante novità, essendosi il giudice avvalso di tecniche neuroscientifiche per lo studio dei processi mnemonici della vittima, al fine di accertare se si fosse effettivamente verificata una violenza sessuale 34 .

Nei fatti, un noto commercialista di Cremona veniva accusato di molestie sessuali da parte di una giovane stagista presso il suo studio ${ }^{35}$. Il giudice per l'udienza preliminare, nel corso del giudizio abbreviato, disponeva una perizia sulla vittima, con il seguente riferimento normativo: "La delicatezza del caso, anche alla luce delle argomentazioni dei difensori che [hanno contestato] la credibilità del racconto della ragazza, portava alla decisione di questo Giudice, all'udienza del 20.1.2011 e quindi al termine della discussione finale, di procedere, ai sensi dell'art. 441 quinto comma c.p.p., a una perizia quale indagine integrativa. Tale accertamento era strutturato secondo la duplice finalità di verificare se la ragazza da un lato avesse dentro di sé il ricordo di quanto aveva ripetutamente narrato e, d'altro lato, quella di verificare se tale evento fosse stato potenziale causa di un danno post-traumatico da stress, soddisfacendo in tal modo non solo un'esigenza di approfondimento ma anche, se del caso, quella di una più precisa valutazione della richiesta di risarcimento formulata dalla parte civile". In questo caso, trovandosi il giudice di fronte a degli indizi equivoci, decideva di avvalersi delle neuroscienze, per verificare attraverso l'Implicit Association Test (IAT) e il Timed Antagonistic Response Alethiometer (TARA), se fosse effettivamente presente nella memoria della vittima una traccia mnestica della violenza sessuale denunciata. Si tratta di tecniche diagnostiche che, come si afferma nella stessa sentenza, "si basano su una valutazione del contenuto della memoria fondata sui tempi di reazione in risposta a frasi che descrivono l'evento autobiografico oggetto della verifica". Ma si chiarisce subito: "al fine di evitare ogni equivoco, che tali metodologie nulla hanno a che vedere con gli antiquati tentativi di verificare la 'sincerità' di un soggetto tramite lie detectors o poligrafi, strumenti che pretenderebbero di fondare la valutazione su grossolani sintomi psico-fisici del periziando". Proseguendo nell'analisi del testo di questa sentenza, si legge: "Premesso che né 
lo I. A.T. né il T.A.R.A., ovviamente, hanno finalità accusatorie ma sono strumenti 'neutri', i risultati della perizia, letti nel contesto generale del processo, hanno offerto un esito di conferma delle dichiarazioni della persona offesa", evincendosi che i risultati della perizia sono stati ritenuti validi, convincendo il giudice, al di là di ogni ragionevole dubbio, della colpevolezza dell'imputato. Secondo quanto affermato dal giudice, sulla base delle dichiarazioni dell'esperto, gli strumenti scientifici utilizzati risponderebbero ai criteri di scientificità, avendo superato "precedenti verifiche e cioè falsificabilità della teoria in senso popperiano e quindi resistenza del metodo a tentativi di smentita, controllo dei lavori pubblicati da parte di revisori qualificati ('peer review'), accettabilità dei limiti di errore, accoglimento da parte della comunità scientifica", giungendosi, quindi, a confermare i criteri di scientificità enucleati dalla sentenza Daubert e sposati dalla giurisprudenza della Cassazione ${ }^{36}$. Nella sentenza in esame, inoltre, si afferma che "altri criteri di giudizio affinati in seguito, quali la 'statura scientifica' dei consulenti, l'impiego precedente delle tecniche in ambiti forensi, l'esclusione di margini di 'soggettività' nella lettura e nell'interpretazione dei risultati, la chiarezza e la semplicità della metodologia usata, risultano ugualmente soddisfatti dalle tecniche usate nella perizia".

La pericolosità sociale e le misure di sicurezza: la Legge n. 81 del 2014 e le sue questioni aperte

"Dal giudizio di pericolosità discende l'applicazione delle misure di sicurezza [...] Si tratta di provvedimenti finalizzati a risocializzare il delinquente, promuovendone la cura e la rieducazione e ponendolo al tempo stesso nella impossibilità di nuocere: il perseguimento di quest'ultimo scopo corrisponde alla specifica esigenza di proteggere la società dagli individui pericolosi" ${ }^{37}$.

Fra le numerose misure di sicurezza previste dal nostro ordinamento rientra il ricovero in Ospedale Psichiatrico Giudiziario (OPG), di cui, di recente, dalla Legge n. 81 del 2014 è stato disposto il superamento a favore delle Residenze per l'Esecuzione delle Misure di Sicurezza (REMS) ${ }^{38}$. Tale intervento legislativo presenta una prima parte incentrata sul superamento degli OPG dal punto di vista strettamente organizzativo, prevedendo:

- la creazione delle residenze per l'esecuzione delle misure di sicurezza (REMS), la quale deve essere necessariamente monitorata relativamente alle attività che le Regioni devono e dovranno porre in essere per mettere effettivamente in funzione queste strutture;

- l'istituzione, presso il Ministero della Salute, di un Organismo volto al coordinamento per il superamento degli OPG;

- la concessione di un termine alle Regioni per rivedere i propri programmi di finanziamento per la riconversione degli OPG;

- la previsione, entro il termine di un mese, di una comunicazione al Ministero della Salute e alla competente Autorità giudiziaria, di programmi di dimissione delle persone attualmente ricoverate negli OPG, a meno che siano presenti ragioni eccezionali che giustifichino la necessità del proseguo del ricovero dei pazienti in cui la pericolosità sociale risulti ancora persistente.

La disciplina delle misure di sicurezza detentive applicabili ai soggetti ritenuti infermi di mente, risulta profondamente modificata dalla seconda parte del provvedimento legislativo in oggetto. All'art. 1, comma 1, lett. b del D.L. n. 52 del 2014, come modificato dalla Legge di conversione n. 81 del 2014, infatti, è previsto che "il giudice dispone nei confronti dell'infermo di mente e del seminfermo di mente l'applicazione di una misura di sicurez$z a$, anche in via provvisoria, diversa dal ricovero in un ospedale psichiatrico giudiziario o in una casa di cura e custodia, salvo quando sono acquisiti elementi dai quali risulta che ogni misura diversa non è idonea ad assicurare cure adeguate e a fare fronte alla sua pericolosità sociale". Il giudice, quindi, dovrà procedere all'applicazione sussidiaria delle misure di sicurezza detentive ai soggetti non imputabili o semi imputabili, solo quando ogni altra misura dovesse risultare inadeguata.

Per quanto, invece, riguarda l'accertamen- 
to della pericolosità sociale, secondo quanto disposto dall'articolo appena citato, "è effettuato sulla base delle qualità soggettive della persona e senza tenere conto delle condizioni di cui all'articolo 133, secondo comma, numero 4, del codice penale. Allo stesso modo provvede il magistrato di sorveglianza quando interviene ai sensi dell'articolo 679 del codice di procedura penale. Non costituisce elemento idoneo a supportare il giudizio di pericolosità sociale la sola mancanza di programmi terapeutici individuali [...] La ratio di una tale novità, risiede, certamente, come già detto, nell 'intento di evitare che situazioni sfavorevoli, quali l'indigenza o l'abbandono, possano influire sul giudizio di pericolosità. Si vuole in altri termini ridurre il peso dei fattori ambientali nella valutazione della pericolosità e restringere l'accertamento di pericolosità alle sole qualità soggettive della persona" 39. Il giudice della cognizione ed il magistrato di sorveglianza, quando devono applicare una misura di sicurezza all'infermo o al seminfermo di mente, devono quindi accertare la sua pericolosità sociale sulla base delle sue qualità soggettive, con esclusione:

1. "delle condizioni di cui all'articolo 133, secondo comma, numero 4 del codice penale" cioè delle sue condizioni di vita individuale, familiare e sociale;

2. della sola assenza di programmi terapeutici individuali.

In pratica si prevede che 1'accertamento della pericolosità sociale, ai fini dell'applicazione delle misure di sicurezza detentive, quali l'O.P.G. (ex art. 222 c.p.) o la C.C.C. (casa di cura e di custodia, ex artt. 219-221 c.p.), sia effettuato esclusivamente sulla base delle qualità soggettive della persona, senza tenere conto delle condizioni di vita individuale, familiare e sociale del reo (art. 133, co. 2 , n. 4 c.p.).

In merito a questa norma in particolare, non sono mancate perplessità riguardo alle possibili conseguenze che discendono dalla stessa, ed in un caso in particolare, tali perplessità hanno assunto la forma di un'ordinanza di rimessione alla Corte Costituzionale. In particolare ci si riferisce all'ordinanza del Tribunale di Sorveglianza di Messina del 16 luglio 2014, la quale ha sollevato la questione di legittimità costituzionale dell'art. 1 della Legge n. 81/2014 nelle parte in cui dispone che l'accertamento della pericolosità sociale nei confronti dell'infermo e del seminfermo di mente deve essere effettuato dal magistrato - sia esso giudice di merito o magistrato di sorveglianza — "sulla base delle qualità soggettive della persona e senza tenere conto delle condizioni di cui all'articolo 133, secondo comma, numero 4, del codice penale", precisando che "non costituisce elemento idoneo a supportare il giudizio di pericolosità sociale la sola mancanza di programmi terapeutici individuali", risultando chiaro, come può leggersi nell'ordinanza, che "dovendo fondare il giudizio prognostico sulla base delle qualità soggettive della persona, ignorando forzatamente $i$ fattori prognostici interdetti dalla normativa denunziata, ancorché acquisiti alla cognizione del fascicolo processuale, la stessa prognosi risulti impossibile o radicalmente alterata - in quanto tale prognosi altro non è che la previsione in chiave probabilistica dei comportamenti che il soggetto potrà assumere proprio nel contesto delle condizioni individuali, familiari, socioassistenziali e sanitarie di cui la norma prescrive di non tener conto - con la conseguenza che sarà ardua, profondamente incerta ed affidata ad un volontarismo giudiziario arbitrario, cognitivamente inadeguato e teleologicamente disorientato, la scelta di se e di quale misura mantenere o adottare e del suo contenuto prescrittivo". 40

La Corte Costituzionale, tuttavia, con la sentenza n. 186 del 24 giugno 2015, ha ritenuto infondata la questione sollevata dal Tribunale di Sorveglianza di Messina, sottolineando che "come emerge dal chiaro tenore letterale del petitum e dagli argomenti sviluppati nell'ordinanza di rimessione, la questione muove dal presupposto che le disposizioni censurate abbiano modificato, relativamente ai 'non imputabili e ai semimputabili', la nozione di pericolosità sociale, che costituisce il presupposto soggettivo delle misure di sicurezza. Infatti nell'ordinanza si sottolinea, tra l'altro, che con la normativa introdotta in sede di conversione del citato decreto-legge sono state effet- 
tuate 'modifiche strutturali di istituti secolari come la pericolosità sociale, disciplinata dalle norme cardinali degli artt. 133 e 203 del codice penale'. Si sarebbe spezzato, 'a livello della prognosi giudiziaria, il rapporto inscindibile tra l'uomo e l'ambiente [...] rinunziando cosi al dinamismo che da tale rapporto scaturisce [e contrastando] le stesse essenziali finalità delle misure di sicurezza sottese al sistema costituzionale'. È su questo presupposto interpretativo che si innestano tutte le numerose censure d'illegittimità costituzionale sopraindicate, ma si tratta di un presupposto errato. Basta leggere la disposizione impugnata per comprendere che le frasi sulle quali si appunta la censura non riguardano la pericolosità sociale come categoria generale, ma si riferiscono più specificamente alla pericolosità che legittima il 'ricovero in un ospedale psichiatrico o in una casa di cura' [...] Ciò posto, è evidente che la disposizione censurata non ha modificato, neppure indirettamente, per le persone inferme di mente o seminferme di mente, la nozione di pericolosità sociale, ma si è limitata ad incidere sui criteri di scelta tra le diverse misure di sicurezza e sulle condizioni per l'applicazione di quelle detentive. L'erroneità del presupposto interpretativo posto a base della questione sollevata dal Tribunale di sorveglianza di Messina ne comporta l'infondatezza in relazione a tutti i residui parametri evocati".

Alla luce di quanto affermato dalla Consulta, il disposto normativo preso in esame risulta pienamente legittimo dal punto di vista del rispetto dei parametri costituzionali. Tuttavia ciò non toglie che vi siano altri profili dai quali poter evincere che, per quanto non incostituzionale, questa precisa disposizione risulta essere inappropriata, avuto riguardo dei profili pratici della sua applicazione in relazione alle difficoltà concrete che sia il giudice che il perito dovranno affrontare in sede di valutazione della pericolosità sociale. Le moderne ricerche scientifiche, infatti, hanno portato a considerare la malattia mentale come necessariamente dipendente da una correlazione di fattori di tipo "bio-psico-sociale", e il prescindere dai fattori contenuti all'art. 133, secondo com- ma, numero 4, c.p., si pone, quindi, in netto contrasto con queste ricerche e, di fatto, con alcuni fra i punti fermi della psichiatria moderna. Tale approccio rischia, inevitabilmente, di riportare alla luce quell'automatismo che tanto si è cercato di scardinare negli ultimi anni, il quale vede nell'atto irrazionale di chi delinque la necessaria presenza di un disturbo mentale, negando la possibilità che alcune azioni siano mosse da ragioni antropologiche e sociali. È innegabile che la nostra personalità, le nostre azioni, sono condizionate dal contesto in cui viviamo, dagli stimoli esterni che riceviamo, dalla possibilità di autodeterminarci e trovare una realizzazione, dall'educazione e formazione. Si può, quindi, forse negare che, così come le azioni positive e altruiste degli esseri umani siano necessariamente legate al nostro background, agli esempi che abbiamo ricevuto, anche le azioni criminose lo siano? Si può realmente prescindere dall'interrogarsi su cosa possa avvenire se un determinato soggetto venga ricollocato nel medesimo contesto sociale nel cui ambito si sono verificati gli input che hanno determinato il suo agire criminoso?

Non si può, inoltre, non evidenziare l'incongruenza tra quanto disposto dalla legge e la prassi che vede la magistratura sollecitare i DSM affinché si adoperino per il reinserimento lavorativo, per una collocazione abitativa dignitosa e anche per eventuali forme di sussidio economico per gli ex pazienti OPG. Occorre, inoltre, tenere presente gli indicatori di pericolosità individuati da Ugo Fornari, che distingue tra indicatori interni, attinenti nello specifico al soggetto rispetto alla sua condizione mentale e quindi alla persistenza o meno della malattia e al suo eventuale sviluppo, nonché alla sua volontà di sottoporsi alle cure, e indicatori esterni che, invece, sono costituiti da elementi di tipo, appunto, sociale ambientale e familiare ${ }^{41}$.

"Con l'attuale norma i cosiddetti 'indicatori esterni' non sono più utilizzabili, e gli indicatori 'interni', sebbene clinicamente logici, non sono mai stati validati a livello statistico, e non si dispone, perciò, di alcun utile strumento evidence-based per effettuare questo tipo di valutazione, il che appare incongruo con un 
SSN che ha come fondamento, invece, la medicina basata sulle evidenze" 42.

L'articolo 1 quater, introdotto dalla Legge 30 maggio 2014 n. 81, di conversione del D.L. 31 marzo n. 52 del 2014, prevede, quale ultima novità che "le misure di sicurezza detentive provvisorie o definitive, compreso il ricovero nelle residenze per l'esecuzione delle misure di sicurezza, non possono durare oltre il tempo stabilito per la pena detentiva prevista per il reato commesso, avuto riguardo alla previsione edittale massima. Per la determinazione della pena a tali effetti si applica l'articolo 278 del codice di procedura penale. Per i delitti puniti con la pena dell'ergastolo non si applica la disposizione di cui al primo periodo".

Alla luce del principio affermato dalla novità normativa appena esposta, risulta opportuno chiedersi come sarà possibile procedere, una volta scaduto il termine massimo della misura, avuto riguardo al fatto che, anche qualora ai sensi del terzo comma dell'art. 232 c.p. nel soggetto sottoposto a misura di sicurezza fosse persistente la pericolosità sociale, non sarebbe possibile riconvertire la libertà vigilata in una misura detentiva. A tal fine risulta fondamentale, inoltre, tenere presente una pronuncia della Cassazione che ritiene sia "illegittimo il provvedimento con cui il tribunale del riesame sostituisca la misura di sicurezza provvisoria del ricovero presso una casa di cura psichiatrica interna al dipartimento dell'amministrazione penitenziaria con altra misura di sicurezza provvisoria presso una struttura comunitaria psichiatrico-residenziale da individuarsi a cura del locale centro di salute mentale, poiché è precluso al giudice applicare misure di sicurezza diverse da quelle previste dalla legge, pena la violazione del principio di legalità, di cui all'art. 25, comma 3, Cost., che sottende una stretta riserva di legge" 43.

Appare evidente, quindi, che il ricorso a case di cura convenzionate e comunità terapeutiche sarebbe illegittimo. Ciò conduce a ritenere come unica possibilità quella di poter eventualmente far ricorso all'applicazione contestuale di una misura non detentiva in strutture "aperte" presenti sul territorio, quali Dipartimenti di igiene mentale, che però potrebbero non essere adatte a questo tipo di assistenza.

L'inserimento di questa previsione ha radicalmente cambiato il principio sotteso alla correlazione tra misura di sicurezza e pericolosità sociale, non essendo più il termine massimo di una misura di sicurezza legato alla pericolosità sociale del soggetto, ma alla pena edittale prevista per il reato commesso.

$\mathrm{Ci}$ si chiede se in questo modo non venga tradito il principio stesso del "doppio binario" posto alla base della differenza tra pena e misura di sicurezza, laddove, se la pericolosità sociale costituisce il presupposto imprescindibile per l'applicazione di una misura di sicurezza, tale presupposto non può, almeno per chi scrive, scomparire una volta accertatane la sussistenza, per essere sostituito da un criterio legato alla durata della pena, come tale afferente all'altro "binario parallelo", ma non sovrapposto a quello della misura di sicurezza.

Senza contare il fatto, che il riferirsi al limite edittale massimo previsto dal Codice per il singolo reato, conduce ad ignorare la valutazione differenziata fatta in sede di giudizio legata alla semiinfermità mentale o totale infermità, alla quale segue una diminuzione della pena o si determina l'esclusione in toto della stessa. Operare in questi termini, di fatto, vanifica l'intento del legislatore di comminare la pena in modo "ragionevolmente" differenziato rispetto al pienamente capace di intendere e di volere, e come minimo, anche laddove si volesse mantenere questo nuovo legame fra misura di sicurezza e durata della pena, questo debba avere ad oggetto, nel caso del semiinfermo di mente, la pena effettivamente comminata in sede di giudizio e, nel caso dell'infermo di mente, il previgente criterio del legame tra misura di sicurezza e persistenza della pericolosità sociale.

Ma a questo punto sorgono spontanee una serie di domande, tenuto conto del fatto, che, una malattia non cessa per effetto di un automatismo giuridico. Cosa succede quando i sintomi della malattia mentale al termine dell'esecuzione della misura di sicurezza risultino tutt'altro che scemati? In che modo si provvederà all'assistenza del malato? Chi si farà carico della sicurezza e della prevenzione? 
Di fatto i soggetti ritenuti ancora affetti da patologia mentale dovranno essere affidati "alle cure degli specialisti, nei presidî ordina$r i$, in assenza di vincoli imposti dall'autorità giudiziaria, avvalendosi magari del dispositivo del Trattamento Sanitario Obbligatorio (TSO), comunque all'interno della posizione di garanzia gravante sugli operatori psichiatrici. Tuttavia, per la clinica psichiatrica le persone non possono essere valutate come 'pericolose' con l'attuale normativa civilistica. Ne deriva che una persona ancora non clinicamente stabilizzata o ritardata mentale, con possibili comportamenti impulsivi, o con delirio cronico strutturato resistente alle terapie e con precedenti di comportamenti antigiuridici contro la persona e un grave disturbo di personalità, al termine della misura determinata dal Codice Penale è da considerarsi libera. Tuttavia, questa persona, specialmente se trattasi di paziente psichiatrico grave, con patologia mentale non stabilizzata, e incapace di dare consenso al trattamento, non necessariamente è nelle condizioni per un TSO, ma non è accettabile che sia lasciato a sé stesso, dal momento che, verosimilmente, si ricadrebbe nell'abbandono di incapace (vedi art. 591 c.p.).

In una schematizzazione di tipo cronologico si potrebbero cosi scandire le entrate (e le uscite) di scena dello psichiatra dal palcoscenico operativo futuro (ma già vigente):

- psichiatra Sİ: per il giudizio di pericolosità sociale all'ingresso;

- psichiatra NO: per la rivalutazione dell'eventuale permanenza della pericolosità sociale perché tanto la temporizzazione interviene per misura di legge;

- psichiatra Sİ: per tutti gli interventi vincolati agli obblighi della posizione di garanzia, ovviamente in ossequio alla legge che impone la gestione dei casi nei DSM, soprattutto con particolare cautela verso coloro che sono a rischio di condotte lesive (presa in carico per la sicurezza della collettività, dunque prevalenza del concetto di 'difesa sociale'sul principio del 'diritto, e appropriatezza, delle cure').

Questa altalena in un quadro normativo privo di una adeguata legge sulla responsabilità professionale. Al termine dei giochi, una sorta di psichiatra-marionetta tirato dai fili delle diversificate necessità di volta in volta subentranti, nonché da altri individuate" 42.

Prendendo, invece, in considerazione il problema opposto, qualora il termine massimo non fosse scaduto e fosse ancora presente pericolosità sociale, ai sensi della Legge 30 maggio 2014 n. 81, si deve procedere alla traslazione dei ricoverati dagli OPG alle REMS.

Tuttavia, è impossibile non considerare che attualmente le REMS non possono ancora garantire le funzioni che la riforma gli ha deferito, e di fatto, nella maggior parte dei casi, risulta difficile, per il momento, giungere al superamento effettivo degli OPG.

L'attuale realtà delle REMS è caratterizzata dal sovrannumero dei pazienti e la presenza di guardie a perimetro delle strutture non sufficiente a garantire la sicurezza all'interno delle strutture, la quale di fatto diventa compito dei medici e operatori sanitari, il cui compito è invece quello della cura e assistenza dei pazienti.

In particolare è indubbio che nessuna delle strutture esistenti presenti i requisiti necessari al fine di far fronte al trattamento altamente specialistico che questi pazienti richiedono. $\mathrm{Si}$ pensi ai casi di pazienti il cui livello di psicopatia sia estremamente elevato e che quindi necessitano di contenimento per il quale queste strutture non sono attrezzate. Questa popolazione di pazienti si presenta particolarmente eterogenea e si pone, quindi, il problema del trattamento differenziato che andrebbe applicato. Si può fare l'esempio di quel sottogruppo di pazienti internati per reati sessuali e stalking, i quali necessiterebbero di strutture specifiche, concepite ad hoc per persone con problemi di questo tipo, che si differenziano sensibilmente dai pazienti psicotici ricoverati nelle REMS.

Inoltre, si evidenzia:

- l'assenza di un piano di intervento condiviso con le altre istituzioni sul problema dei senza fissa dimora e di tutti quei pazienti che non potranno essere ricollocati in famiglia anche dopo la fine del percorso terapeutico;

- l'assenza della previsione di una procedura condivisa con le prefetture e con le altre istituzioni coinvolte per la gestione dei pazien- 
ti extra-comunitari senza permesso di soggiorno, considerata l'estrema difficoltà ad effettuare un rimpatrio degli stessi;

- la mancanza di un procedimento rapido per l'amministrazione di sostegno;

- la mancanza di un istituto a tutela dei pazienti valutati come non in grado di stare in giudizio.

Affinché si riesca ad inquadrare il raggiungimento della sicurezza, come il risultato di un sistema di relazioni reciproche tra il paziente e il suo ambiente, nel quale venga eseguito un processo di stabilizzazione e ripresa, è necessario avvalersi di regolamentazioni che consentano di realizzare un equilibrio tra la salvaguardia dei pazienti e del personale e la costruzione di un ambiente solido e sicuro, che possa garantire davvero lo svolgimento di un percorso terapeutico. La sicurezza di una struttura operativa può essere garantita avvalendosi dell'ausilio di:

- tecnologie specifiche e meccanismi per il controllo dei perimetri interni ed esterni, meccanismi di sicurezza e le tecnologie, quali serrature elettroniche, videosorveglianza e altre barriere fisiche presenti nell'unità e il servizio nel suo complesso, non essendo possibile prescindere dal definire in modo chiaro e presidiabile i perimetri interni ed esterni destinati ai pazienti o ad essi interdetti, creando, così, le condizioni per evitare comportamenti auto ed etero aggressivi dei pazienti e proteggere il personale;

- procedure operative tempestive, corrette e coerenti;

- livello adeguato di manutenzione ordinaria e straordinaria dello stabile degli impianti e dalle condizioni delle attrezzature e degli arredi utilizzati;

- un alto livello di consapevolezza relazionale quale "personale conoscenza e comprensione del contesto ambientale del singolo paziente e delle relazioni e dinamiche che attraversano il contesto che si traduce nella capacità di convertire tali informazioni e vissuti esperienziali in risposte adeguate e cure" 44.

Non può non considerarsi, infine, che spesso i pazienti devono rimanere ricoverati per lunghi periodi di tempo, il che determina l'esi- genza di spazi che riescano a far fronte, il più possibile, a quelle che sono le esigenze sociali, terapeutiche e di cura personale dell'essere umano, al fine di garantirne la dignità. "Per esempio nelle REMS andrebbe previsto un bar, un luogo di culto per le diverse religioni, un luogo per la de-escalation, una sala per ricevere le cure alla persona come interventi podologici, parrucchiere etc. L'Italia potrà gestire questa opportunità storica di un trattamento innovativo solo ispirandosi alla scienza e ai dati della letteratura, l'improvvisazione in questa situazione sarebbe una forma di razionalità negata" 45 .

Infine, una delle questioni applicative più critiche che interessano la Legge n. 81 del 2014, attiene all'ipotesi prevista dall'art. 206 del codice penale: "Durante la istruzione o il giudizio, può disporsi che il minore di età, o l'infermo di mente, o l'ubriaco abituale, o la persona dedita all'uso di sostanze stupefacen$t i$, o in stato di cronica intossicazione prodotta da alcool o da sostanze stupefacenti, siano provvisoriamente ricoverati in un riformatorio o in un ospedale psichiatrico giudiziario, o in una casa di cura e di custodia. Il giudice revoca l'ordine, quando ritenga che tali persone non siano più socialmente pericolose. Il tempo dell'esecuzione provvisoria della misura di sicurezza è computato nella durata minima di essa". Come precedentemente visto, infatti, all'art. 1, comma 1, lett. b del D.L. n. 52 del 2014, come modificato dalla Legge di conversione n. 81 del 2014, è previsto che "il giudice dispone nei confronti dell'infermo di mente e del seminfermo di mente l'applicazione di una misura di sicurezza, anche in via provvisoria, diversa dal ricovero in un ospedale psichiatrico giudiziario o in una casa di cura e custodia, salvo quando sono acquisiti elementi dai quali risulta che ogni misura diversa non è idonea ad assicurare cure adeguate e a fare fronte alla sua pericolosità sociale".

Non si può non notare, tuttavia, che, nella pratica, nel caso dell'applicazione di misure di sicurezza provvisorie, procedere solo in via sussidiaria alla scelta del ricovero in ospedale psichiatrico giudiziario o in casa di cura e custodia risulta non poco difficoltoso. 
Il nostro ordinamento, nell'intervento nei confronti di persone pericolose, appronta, infatti, due sostanziali strumenti: le misure cautelari e le misure di sicurezza. Entrambe le misure hanno la finalità comune di evitare che la persona rimanga in stato di libertà, se sussiste la concreta e qualificata probabilità che da libera possa commettere reati, e quindi possa porre a repentaglio la sicurezza della collettività. Per il resto, però, esse si diversificano profondamente.

La misura cautelare richiede un giudizio per gravi indizi di colpevolezza. Tuttavia, la valutazione della presenza dei gravi indizi di colpevolezza, presuppone che il giudice sia in una fase antecedente l'accertamento della responsabilità, in condizione di poter dire con elevatissima probabilità che l'individuo ha tenuto una certa condotta; che quella condotta sia di reato e quindi riconducibile ad una delle categorie di reato per le quali la misura cautelare può essere applicata e che si possa valutare la colpevolezza della persona, che è una categoria composta da di tanti momenti. Il primo di questi è la rimproverabilità del fatto a chi lo ha commesso, la quale muove dalla certezza che la persona che l'ha commesso si è liberamente determinata a commetterlo, ovvero lo ha commesso sapendo di tenere una condotta antisociale e con quella sufficiente coscienza e volontà che integra poi il dolo. La colpevolezza richiede, quindi, che una persona possa essere rimproverata per aver tenuto quella condotta perché ha scelto di tenerla liberamente, quindi senza alcuna costrizione non solo da parte di terzi ma anche senza impulso della malattia. Nel momento in cui il giudice accerta la ricorrenza in termini di elevata probabilità di queste condizioni, applica la misura cautelare, la quale può essere la custodia in carcere e, se la persona è inferma di mente, in casa di cura. L'articolo 286 c.p.p., concernente le misure cautelari, stabilisce che "se la persona da sottoporre a custodia cautelare si trova in stato di infermità di mente che ne esclude o ne diminuisce grandemente la capacità di intendere o di volere, il giudice, in luogo della custodia in carcere, può disporre il ricovero provvisorio in idonea struttura del servizio psichiatrico ospedaliero, adottando i provvedimenti necessari per prevenire il pericolo di fuga. Il ricovero non può essere mantenuto quando risulta che l'imputato non è più infermo di mente. Si applicano le disposizioni dell'articolo 285 commi 2 e 3".

Nell'eventualità in cui, tuttavia, manchino gli elementi idonei per formulare un giudizio di gravi indizi di colpevolezza, che è il presupposto per applicare la misura cautelare, l'unica possibilità del giudice, quando deve valutare la pericolosità di un individuo che ha un problema di salute mentale, problema che incide a tal punto sulla capacità da escluderla, è purtroppo l'applicazione provvisoria di una misura di sicurezza. Le case di cura e custodia di cui parla il codice penale, tra l'altro, non sono delle sezioni specializzate degli OPG, ma sono gli OPG stessi. Di fatto, quindi, la casa di cura e custodia e l'ospedale psichiatrico giudiziario, oggi REMS, sono sostanzialmente la stessa cosa. Posto che la casa di cura e custodia non è niente di diverso dall'ospedale psichiatrico giudiziario, l'unica possibilità è applicare la libertà vigilata, che è una misura di sicurezza non detentiva, e si può applicare qualunque sia il tipo di reato e qualunque sia il grado di incapacità della persona. Se infatti, precedentemente, la libertà vigilata poteva essere disposta soltanto per ipotesi meno gravi, la Corte Costituzionale con la sentenza del 29 novembre 2004, n.367, ha disposto che "l'illegittimità costituzionale dell'art. 206 del codice penale (Applicazione provvisoria delle misure di sicurezza), nella parte in cui non consente al giudice di disporre, in luogo del ricovero in ospedale psichiatrico giudiziario, una misura di sicurezza non detentiva, prevista dalla legge, idonea ad assicurare alla persona inferma di mente cure adeguate e a contenere la sua pericolosità sociale".

\section{Conclusioni}

Non sono rare le affermazioni di chi sostiene che lo psichiatra che si appresta ad effettuare il giudizio di pericolosità sociale abbia le stesse probabilità di successo di chi si affida al lancio di una moneta per prendere una 
decisione. ${ }^{46}$ Sono molti, infatti, gli psichiatri che contestano la scientificità del concetto di pericolosità sociale, in quanto, questo, è "una normazione giuridica e pertanto non ha asilo in campo medico" 47 e sembrerebbero, quindi, mancare gli strumenti scientifici "in grado di offrirci dati attendibili di fronte a una realtà composita e complessa, in cui la componente 'persona umana' con la sua storia di vita, le sue esperienze, la sua soggettività, le sue imprevedibilità, è tutt'altro che una variabile di secondo piano" 48. Tali considerazioni hanno condotto la psichiatria, o comunque molti esponenti di questa, a ritenere che l'accertamento della pericolosità sociale dei soggetti ritenuti non imputabili non possa essere di sua competenza.

Non si può fare a meno di notare, tuttavia, anche e soprattutto alla luce dei fattori da prendere in considerazione nel giudizio di pericolosità sociale, che, con tutta evidenza, vi sono degli aspetti tecnici che non possono essere valutati dal solo giudice, il quale, necessariamente, non ha le competenze adeguate, trattandosi anche di dover effettuare un inquadramento nosografico del soggetto. Risulta, quindi, difficile, che vi possa essere una figura più idonea dello psichiatra a valutare la pericolosità sociale dei soggetti non imputabili e a valutare gli effetti dei disturbi psichici sulla probabilità di futura recidiva.

Piuttosto, sembrerebbe che, affinché l'istituto della pericolosità sociale possa muoversi a servizio e tutela del singolo quanto della collettività, rispettandosi le finalità delle misure di sicurezza, sia necessaria una stretta collaborazione tra giustizia e psichiatria, nella quale l'attuazione di un programma individualizzato di trattamento terapeutico e un dialogo continuo tra gli operatori di giustizia e della sanità, in relazione all'assistenza, al contesto familiare e relazionale del soggetto, in funzione del suo reinserimento in società, siano i veri protagonisti.

Non ci si può dimenticare quale sia la finalità della misura di sicurezza e perché si sia deciso che la pena e la misura di sicurezza debbano lavorare su due binari differenti, anche se complementari. Le misure di sicurezza, come ampiamente detto, sono concepite come strumenti di recupero che al tempo stesso possano garantire l'incolumità della società. Il recupero dei pazienti sottoposti a misure di sicurezza detentive, non può infatti prescindere dal tentativo di riadattare questi soggetti, dal tentativo di renderli partecipi di una società della quale hanno necessariamente fatto parte, così come è impossibile che la finalità di recupero venga perseguita correttamente se la durata delle misure di sicurezza continuerà ad essere legata al limite edittale massimo della pena previsto dal codice, così come da Legge n. 81 del 2014, invece che all'effettiva persistenza o assenza della pericolosità sociale.

L'unico modo, tuttavia, perché questo si verifichi è che questo tentativo possa fondarsi su degli strumenti adeguati, sull'ausilio di trattamenti terapeutici opportuni, nell'ambito di strutture sanitarie che possano svolgere davvero il compito a loro destinato, senza mai dimenticare l'esigenza del raggiungimento di un equilibrio tra la tutela di questi individui, e quindi la tutela del singolo, e la tutela della collettività.

Prima ancora che un individuo possa essere pronto a reinserirsi in società, ammesso che ci riesca, è necessario che possa sentirsi parte dell'ambiente in cui deve essere curato e trattato. Così come è indispensabile che, laddove, il suo percorso in misura di sicurezza detentiva sia da ritenersi finito, o non opportuno, vi sia una rete assistenziale, adeguatamente differenziata, in grado di porsi come supporto in caso di necessità. Sarà quindi necessario:

- "facilitare la comunicazione tra gli operatori della giustizia e quelli della sanità, dell'assistenza e il contesto familiare e relazionale del soggetto, in funzione delle possibili forme di intervento;

- utilizzare l'elaborato peritale anche pe fornire indicazioni terapeutiche che servano al periziato; $[. .$.

- insistere per ottenere una diversificazione degli ambiti prevalenti di competenza socio sanitaria nel caso delle psicosi e dei disturbi gravi di personalità; socio -terapeutica nel caso dei disturbi di personalità non complicati; 
- ottenere che questi ultimi seguano percorsi sanzionatori, clinici e riabilitativi diversificati rispetto a quelli degli psicotici e dei soggetti affetti da disturbi di personalità, attraverso l'utilizzazione di strutture differenziate per autori di reato psicotici rispetto agli autori di reato affetti da disturbi della personalità;

- operare affinché ogni tipo di intervento sia tempestivamente adottato, sulla base delle indicazioni cliniche fornite dai periti; [...]

- insistere affinché vengano organizzate (o implementate) sul territorio strutture a forte connotazione riabilitativa per malati di mente autori di reato che possono usufruire nella fase della cognizione degli arresti domiciliari e nella fase della esecuzione di tutti quegli interventi "protetti" che consentano la prosecuzione del trattamento comunque iniziato e la verifica della stabilizzazione del quadro psichico in vista di una loro definitiva uscita dal circuito giudiziario" 49.

Per quanto attiene, in particolare, all'aspetto terapeutico, considerato l'innovativo supporto delle neuroscienze in tema di verifica dell'imputabilità, circoscrivere la possibilità di avvalersi di queste scienze solo in tale ambito rischierebbe di essere riduttivo, dato l'importante ausilio che queste potrebbero fornirci per poter individuare nuovi percorsi terapeutici. Si sarebbero individuati "- secondo componenti accreditati nella comunità scientifica internazionale - dei fattori causali rilevanti per l'origine ed il successivo sviluppo di alcune patologie mentali", iniziandosi ad intravedere la concreta opportunità di curare e non solo arginare i sintomi delle malattie, rimuovendone le cause. "Di qui si prospettano - ad opera di una parte emergente della comunità scientifica - programmi biomedici radicali: si ipotizza, infatti - esprimendosi in via assolutamente sommaria - che, intervenendo sul patrimonio genetico della mente umana si possano correggere strutturalmente le patologie mentali, ovvero, si possa correggere qualsivoglia devianza psichica. Per cui - per quanto è ora di nostro interesse - $i$ trattamenti psichiatrici e la disciplina giuridica, riferiti a coloro che presentano compor- tamenti psichicamente devianti, muterebbero radicalmente" 50 .

La Legge n. 81 del 2014 ha segnato profondamente questo tema, rendendolo quanto mai attuale, mediante la volontà di superamento degli Ospedali Psichiatrici Giudiziari, attraverso l'individuazione di un'alternativa nelle REMS, nel tentativo di creare un supporto normativo in grado di poter circoscrivere il più possibile il ricorso a misure di sicurezza detentive, confinandole a un ruolo sussidiario.

Pretendere che la formulazione del giudizio di pericolosità sociale psichiatrica debba prescindere dalla valutazione degli elementi sociali, familiari e ambientali del soggetto in esame, si pone in netto contrasto con le più recenti teorie psichiatriche e criminologiche in materia, escludendo una fetta troppo importante di un puzzle di cui già di per sé è difficile mettere insieme i pezzi.

Al tempo stesso ancorare la durata delle misure di sicurezza al limite edittale massimo della pena prevista per il reato commesso, confonde inevitabilmente i piani di applicazione dell'impianto del "doppio binario" penamisura di sicurezza, che ha sempre costituito le fondamenta di questa materia, sottraendo certezza e risorse temporali alle importanti necessità di cura di cui molto spesso gli autori di reato affetti da malattie psichiatriche hanno bisogno, determinando, di fatto, la perdita della possibilità di questi individui di essere trattati adeguatamente per tutto il tempo necessario, il quale quasi mai potrà corrispondere a quanto previsto dal codice penale in relazione al reato da loro commesso.

Le REMS sono senz'altro un primo passo, necessario, verso il superamento degli OPG, ma, affinché possano realmente costituire una valida alternativa a questi, necessitano di ancora troppe risorse per essere adeguate e pronte alla gestione delle problematiche che interessano i pazienti psichiatrici autori di reato. Le risorse da investire in questo ambito devono essere riconsiderate alla luce della fondamentale esigenza di contemperare la finalità di tutela del diritto alla salute del singolo e del diritto alla sicurezza della collettività.

Soltanto quando in queste strutture il per- 
sonale sanitario verrà messo realmente in condizione di svolgere il proprio lavoro di assistenza ai pazienti e non di coercizione e gendarmeria, sarà possibile ottenere buoni risultati riabilitativi dei soggetti in esse ricoverati, raggiungendo l'obiettivo di rendere il più breve possibile il ricovero di questi soggetti nelle REMS, ottimizzando le risorse ed evitando il più possibile gli sprechi, al servizio del singolo e della collettività, sulla base di un continuo e costruttivo dialogo tra giustizia e sanità, nella continua ricerca di un equilibrio, oggi ancora troppo precario.

\section{Riassunto}

Il nostro ordinamento, nell'intervento nei confronti di soggetti pericolosi, prevede due sostanziali strumenti: le misure cautelari e le misure di sicurezza detentive. Le misure di sicurezza detentive sono concepite come strumenti di recupero che al tempo stesso possano garantire l'incolumità della società. La Legge n. 81 del 2014 ha segnato profondamente questo tema, rendendolo quanto mai attuale, mediante la volontà di superamento degli Ospedali Psichiatrici Giudiziari, attraverso l'individuazione di un'alternativa nelle REMS, nel tentativo di creare un supporto normativo in grado di poter circoscrivere il più possibile il ricorso a misure di sicurezza detentive, confinandole ad un ruolo sussidiario.

\section{References}

1. Feuerbach L. Revision der Grundsätze und Grundbegriffe des positiven peinlichen Rechts. Erfurt: Chemnitz; 1880. p. 356-66.

2. Padovani T. La pericolosità sociale sotto il profilo giuridico. In: Ferracuti F. Trattato di criminologia, medicina criminologica e psichiatria forense: psichiatria forense generale e penale. Milano: Giuffrè; 1990. p. 325.

3. Corte Costituzionale, sentenza n.139, 27 agosto 1982

4. Corte Costituzionale, sentenza n. 249, 15 luglio 1983.

5. Legge n. 663/1986, art. 31, $1^{\circ}$ comma.

6. Cassazione Sezioni Unite, sentenza n. 9163, 8 marzo 2005.

7. Tribunale Napoli sez. VI, sentenza n. 15963, 3 dicembre 2014.

8. Marzano F. Gli ultimi approdi della giurisprudenza di legittimità sul vizio di mente. In: Cipolla $\mathrm{S}$, Bana $\mathrm{A}$. Crimini, criminali e malattia mentale: scienze giuridicopenali e scienze empirico-sociali a confronto. Bruxelles: Bruylant; 2007. p. 13.

9. Bertolino M. Commento alla sentenza delle Sezioni Unite n. 9163 Raso. Dir Pen e processo 2005;7:119.

10. Fidelbo G. Le Sezioni Unite riconoscono rilevanza ai disturbi di personalità. Cass Pen 2005;6:1873.

11. Colombo C, Zanardi R, Smeraldi E, Bruno F, Mastronardi V, Bianchi A, et al. Ricerche Cliniche, testistiche e tecniche nella pratica psichiatrica - forense e criminologica. In: Psichiatria Forense, criminologia ed etica psichiatrica, II edizione. Elsevier-Masson; 2010. p. 110.

12. Ashburner J. Computational anatomy with the SPM software. Magn Reson Imaging 2009;27:1163-74.
13. Voets NL, Hough MG, Douaud G, Matthews PM, James A, Winmill L, et al. Evidence for abnormalities of cortical development in adolescent-onset schizophrenia. Neuroimage 2008;43:665-75.

14. Terrazas A, McNaughton BL. Brain growth and the cognitive map. Proc Natl Acad Sci USA 2000;97:4414-4416.

15. Good CD, Johnsrude IS, Ashburner J, Henson RN, Friston KJ, Frackowiak RS. A Voxel-Based Morphometric Study of Ageing in 465 Normal Adult Human Brains. Neuroimage 2001;14:21-36.

16. Ashburner J, Friston KJ. Voxel-Based Morphometry The Methods. NeuroImage 2000;11:805-821.

17. Kawasaki Y, Suzuki M, Kherif F, Takahashi T, Zhou SY, Nakamura K, et al. Multivariate voxel-based morphometry successfully differentiates schizophrenia patients from healthy controls. Neuroimage 2007;34:235-242.

18. Cadoret RJ, Langbehn D, Caspers K, Troughton EP, Yucuis R, Sandhu HK, et al. Associations of the serotonin transporter promoter polymorphism with aggressivity, attention deficit, and conduct disorder in an adoptee population. Compr Psychiatry 2003;44:88-101.

19. Grigorenko EL, De Young CG, Eastman M, Getchell M, Haeffel GJ, Klinteberg Ba, et al. Aggressive behavior, related conduct problems, and variation in genes affecting dopamine turnover. Aggress Behav 2010;36:158-76.

20. Craig IW, Halton KE. Genetics of human aggressive behaviour. Hum Genet 2009;126:101-13.

21. Craig IW. The importance of stress and genetic variation in human aggression. Bioessays 2007;29:227-36.

22. Pavlov KA, Chistiakov DA, Chekhonin VP. Genetic determinants of aggression and impulsivity in humans. J Appl Genet. 2012;53:61-82.

23. Kerman IA, Clinton SM, Bedrosian TA, Abraham AD, Rosenthal DT, Akil H, et al. High novelty-seeking predicts aggression and gene expression differences within defined serotonergic cell groups. Brain Res 2011;1419:34-45.

24. Kim-Cohen J, Caspi A, Taylor A, Williams B, Newcombe $\mathrm{R}$, Craig IW, et al. MAOA, maltreatment, and gene-environment interaction predicting children's mental health: new evidence and a meta-analysis. Mol Psychiatry 2006;11:903-913.

25. Tielbeek JJ, Karlsson Linnér R, Beers K, Posthuma D, Popma A, Polderman TJ. Meta-analysis of the serotonin transporter promoter variant (5-HTTLPR) in relation to adverse environment and antisocial behavior. Am J Med Genet B Neuropsychiatr Genet 2016;171:748-60.

26. Fabbri C, Cimino L, Serretti A. Genetica dei comportamenti impulsivo-aggressivi: possibile applicazione in psichiatria forense? Rass It Crim 2013;11:34-51.

27. Corte di Cassazione, sentenza n. 45351,11 novembre 2013.

28. Corte di Cassazione, sentenza n.43786, 17 settembre 2010.

29. Corte di Cassazione, sentenza n. 36080, 7 settembre 2015.

30. Corte d'Assise d'Appello di Trieste, sentenza n. 5, 18 settembre 2009.

31. Capra G. Le neuroscienze e la genetica molecolare nella valutazione della capacità di intendere e di volere. Psicologia giuridica 2009.

32. Tribunale di Como, sentenza n.536, 20 maggio 2011.

33. Terracina D. Neuroscienze: lo studio della morfologia del cervello determinante nello stabilire il vizio parziale di mente. In: Guida al Diritto 2012. p. 63.

34. Tribunale di Cremona, sentenza n. 109, 19 luglio 2011.

35. Santuosso A, Bottalico B. Neuroscienze e genetica comportamentale nel processo penale italiano. Casi e prospettive. Ras It Crim 2013. p. 76.

36. Bertolino M. L'imputabilità penale fra cervello e mente. Riv It Med Leg 2012;3:922-939. 
37. Dell'Osso G. Capacità a delinquere e pericolosità sociale. Milano: Giuffrè; 1985. p. 93.

38. Legge n.81/2014.

39. Caputo A. La pericolosità sociale, vecchie esigenze e nuove prospettive alla luce della legge 30 Maggio 2014, n. 81. Roma: Aracne Editrice; 2015. p. 135.

40. Bianchetti R. Sollevata questione di legittimità costituzionale in merito ai nuovi criteri di accertamento della pericolosità sociale del seminfermo di mente - Tribunale di Sorveglianza di Messina, ordinanza del 16 luglio 2014. Diritto Penale Contemporaneo 2014.

41. Fornari U. Trattato di Psichiatria Forense, V edizione. Roma: Utet giuridica; 2013. p. 219-21.

42. Ferracuti S. Come cureremo l'ampio spettro di pazienti con gravi malattie mentali autori di reato? La Legge n. 81/2014: limiti e problematiche. Riv Psichiatr 2015;50:103-9.

43. Corte di Cassazione penale sez. V, sentenza n. 9656, 08 gennaio 2010.

44. Perini R. I requisiti strutturali e la pianificazione del livello di sicurezza nella progettazione di Strutture Resi- denziali Psichiatriche; 2011 [Internet]. Available from: http://ita.calameo.com/books/000718568b5ae5ff6138e [cited 2016, Sep 13].

45. Ferracuti S, Nicolò G, Perini R. OPG: Il futuro sono le Rems? Quotidiano Sanità 2014.

46. Ennis B, Litwak R. Psichiatry and the presumption of expertise: flippings the coins in the courtroom. California Law Review 1974;62:693.

47. Manacorda A. Pericolosità sociale e determinazione della durata minima del ricovero in O.P.G. Foro It 1987;2: 106.

48. Fornari U. Attualità in tema di pericolosità sociale psichiatrica. Relazione a congresso "Pericolosità sociale e società pericolosa", Aversa; 6-8 novembre 2008.

49. Fornari U. Trattato di Psichiatria Forense, V edizione. Roma: Utet giuridica; 2013. p. 222-4.

50. Rossini G. Il controllo pubblico sulla mente: dai trattamenti psichiatrici obbligatori alle neuroscienze. Altalex; 2015 [Internet]. Available from: www.altalex.com/documents/news/2015/11/19/controllo-pubblico-sulla-mentedai-tso-alle-neuroscienze [cited 2016, Sep 13].

Conflicts of interest.-The authors certify that there is no conflict of interest with any financial organization regarding the material discussed in the manuscript.

Manuscript accepted: September 7, 2016. - Manuscript received: August 5, 2016.



